\title{
Errata: Changes of collagen and nicotinamide adenine dinucleotide in human cancerous and normal prostate tissues studied using native fluorescence spectroscopy with selective excitation wavelength
}

\author{
Yang Pu \\ City College of the City University of New York \\ Institute for Ultrafast Spectroscopy and Lasers \\ Departments of Electrical Engineering \\ 160 Convent Avenue \\ New York, New York 10031
}

\section{Wubao Wang \\ Guichen Tang}

Robert R. Alfano

City College of the City University of New York

Institute for Ultrafast Spectroscopy and Lasers

Department of Physics

160 Convent Avenue

New York, New York 10031

[DOI: $10.1117 / 1.3483452]$

This article [J. Biomed. Opt. 15, 047008 (2010)] was originally published online on 23 July 2010 with two errors in the author list. The author listed as "Wubao B. Wang" should have been listed as "Wubao Wang" and "Guichen C. Tang" should have been listed as "Guichen Tang."

All versions of the article were corrected on 30 July 2010. 